\title{
Multiparametric ${ }^{18}$ F-FDG PET/MRI of the Breast: Are There Differences in Imaging Biomarkers of Contralateral Healthy Tissue Between Patients With and Without Breast Cancer?
}

\author{
Doris Leithner ${ }^{1-3}$, Thomas H. Helbich ${ }^{3}$, Blanca Bernard-Davila ${ }^{4}$, Maria Adele Marino ${ }^{1,5}$, Daly Avendano ${ }^{1,6}$, \\ Danny F. Martinez ${ }^{1}$, Maxine S. Jochelson ${ }^{1}$, Panagiotis Kapetas ${ }^{3}$, Pascal A.T. Baltzer ${ }^{3}$, Alexander Haug ${ }^{7,8}$, \\ Marcus Hacker ${ }^{7}$, Yasemin Tanyildizi ${ }^{9}$, Elizabeth A. Morris ${ }^{1}$, and Katja Pinker ${ }^{1,3}$ \\ ${ }^{1}$ Department of Radiology, Breast Imaging Service, Memorial Sloan Kettering Cancer Center, New York, New York; ${ }^{2}$ Department \\ of Diagnostic and Interventional Radiology, University Hospital Frankfurt, Frankfurt, Germany; ${ }^{3}$ Division of Molecular and \\ Gender Imaging, Department of Biomedical Imaging and Image-Guided Therapy, Medical University of Vienna, Vienna, Austria; \\ ${ }^{4}$ Department of Epidemiology and Biostatistics, Memorial Sloan Kettering Cancer Center, New York, New York; ${ }^{5}$ Department of \\ Biomedical Sciences and Morphologic and Functional Imaging, University of Messina, Messina, Italy; ${ }^{6}$ Department of Breast \\ Imaging, Breast Cancer Center TecSalud, ITESM Monterrey, Nuevo Leon, Mexico; ${ }^{7}$ Division of Nuclear Medicine, Department of \\ Biomedical Imaging and Image-Guided Therapy, Medical University of Vienna, Vienna, Austria; ${ }^{8}$ Christian Doppler Laboratory \\ for Applied Metabolomics, Medical University of Vienna, Vienna, Austria; and ${ }^{9}$ Department of Neuroradiology, University Medical \\ Center of the Johannes Gutenberg-University, Mainz, Germany
}

The rationale was to assess whether there are differences in multiparametric ${ }^{18} \mathrm{~F}-\mathrm{FDG}$ PET/MRI biomarkers of contralateral healthy breast tissue in patients with benign and malignant breast tumors. Methods: In this institutional review board-approved prospective single-institution study, 141 women with imaging abnormalities on mammography or sonography (BI-RADS 4/5) underwent combined ${ }^{18} \mathrm{~F}-\mathrm{FDG}$ PET/MRI of the breast at $3 \mathrm{~T}$ with dynamic contrastenhanced $\mathrm{MRI}$, diffusion-weighted imaging, and the radiotracer ${ }^{18} \mathrm{~F}-\mathrm{FDG}$. In all patients, the following imaging biomarkers were recorded for the contralateral (tumor-free) breast: breast parenchymal uptake (BPU) (from ${ }^{18} \mathrm{~F}-\mathrm{FDG} P E T$ ), mean apparent diffusion coefficient (from diffusion-weighted imaging), background parenchymal enhancement (BPE), and amount of fibroglandular tissue (FGT) (from MRI). Appropriate statistical tests were used to assess differences in ${ }^{18} \mathrm{~F}-\mathrm{FDG}$ PET/MRI biomarkers between patients with benign and malignant lesions. Results: There were 100 malignant and 41 benign lesions. BPE was minimal in 61 patients, mild in 56, moderate in 19, and marked in 5. BPE differed significantly $(P<0.001)$ between patients with benign and malignant lesions, with patients with cancer demonstrating decreased BPE in the contralateral tumorfree breast. FGT approached but did not reach significance $(P=$ $0.055)$. BPU was 1.5 for patients with minimal BPE, 1.9 for mild BPE, 2.2 for moderate BPE, and 1.9 for marked BPE. BPU differed significantly between patients with benign lesions (mean, 1.9) and patients with malignant lesions (mean, 1.8) $(P<0.001)$. Mean apparent diffusion coefficient did not differ between groups $(P=0.19)$. Conclusion: Differences in multiparametric ${ }^{18} \mathrm{~F}-\mathrm{FDG}$ PET/MRI biomarkers, obtained from contralateral tumor-free breast tissue, exist between patients with benign and patients with malignant breast tumors. Contralateral BPE, BPU, and FGT are decreased in breast cancer patients and may potentially serve as imaging biomarkers for the presence of malignancy.

Received Apr. 19, 2019; revision accepted May 16, 2019.

For correspondence or reprints contact: Katja Pinker, Department of Radiology/ Breast Imaging Service, Memorial Sloan Kettering Cancer Center, 300 E 66th St., 7th Floor, New York, NY 10065.

E-mail: pinkerdk@mskcc.org

Published online Jun. 28, 2019.

COPYRIGHT (c) 2020 by the Society of Nuclear Medicine and Molecular Imaging.
Key Words: ${ }^{18} \mathrm{~F}-\mathrm{FDG}$ PET/MRI; breast cancer; dynamic contrastenhanced MRI; diffusion-weighted imaging; imaging biomarker

J Nucl Med 2020; 61:20-25

DOI: 10.2967/jnumed.119.230003

B reast cancer is the most common cancer in women in the United States and, despite advances in early detection and treatment, accounts for approximately 40,000 deaths per year (1). Early detection remains key to improved prognosis and survival. Screening mammography has decreased the mortality for breast cancer by $30 \%$, but its sensitivity is limited $(\sim 70 \%)$ and is decreased in women with dense breasts $(2,3)$. Such shortcomings warrant further refinements in breast cancer screening modalities and the identification of imaging biomarkers to enable risk-adapted screening and guide risk-reduction strategies in clinical practice.

MRI is the most sensitive test for breast cancer detection, outperforming mammography and sonography. Adjunct screening with breast MRI is recommended for women at high $(>20 \%)$ lifetime risk of breast cancer (4-6), and recently the American College of Radiology (ACR) issued a similar recommendation for its use in women at intermediate $(>15 \%)$ lifetime risk (7). Additionally, there is evidence that women at average cancer risk might also benefit from screening MRI (8). MRI provides not only morphologic and functional information on breast tumors but also insight into the amount of fibroglandular tissue (FGT) and its physiologic activity, that is, background parenchymal enhancement (BPE) $(9,10)$. Initial results already indicate that $\mathrm{BPE}$ and to some extent FGT, which is equivalent to breast density in mammography $(11,12)$, are increased in high-risk breast cancer patients $(11,13)$. To date, multiparametric MRI of the breast including dynamic contrast-enhanced MRI and diffusion-weighted imaging with apparent diffusion coefficient (ADC) mapping has been implemented into the clinical routine and provides additional functional information on breast tissue (14). 
However, whereas it has been demonstrated that ADCs in breast tumors are an imaging biomarker for tumor grade, invasiveness, and receptor status $(15,16)$, little is known about the significance of ADCs in healthy breast tissue.

Like MRI, PET using the radiotracer ${ }^{18} \mathrm{~F}-\mathrm{FDG}$ provides information on tissue glucose metabolism and, hence, the physiologic activity of breast parenchyma (17). FGT shows varying degrees of ${ }^{18} \mathrm{~F}-\mathrm{FDG}$ uptake, that is, breast parenchymal uptake (BPU), which correlates with both BPE and FGT $(17,18)$ and therefore might also serve as another important imaging biomarker in breast cancer.

Hybrid PET/MRI scanners, now being increasingly used (19-21), can simultaneously assess and spatiolongitudinally monitor these multiple imaging biomarkers and could therefore significantly contribute to risk-adapted screening and risk-reduction strategies in clinical practice. The aim of our study was to assess whether there may be differences in multiparametric ${ }^{18} \mathrm{~F}$-FDG PET/MRI biomarkers of contralateral healthy breast tissue between patients with benign and patients with malignant breast tumors.

\section{MATERIALS AND METHODS}

\section{Patients}

The institutional review board/ethics committee of the Medical University of Vienna approved this prospective single-institution study and retrospective data analysis, and all subjects gave written informed consent. From December 2009 to November 2014, 191 consecutive patients who fulfilled the following criteria were included in this study: at least $18 \mathrm{y}$ old, not pregnant, not breastfeeding, imaging abnormality on mammography or sonography (Breast Imaging Reporting and Data System [BI-RADS] 4, suspicious abnormality; 5, highly suggestive for malignancy), and no contraindications to MRI or contrast agents. All patients underwent combined multiparametric ${ }^{18} \mathrm{~F}-$ FDG PET/MRI of the breast at $3 \mathrm{~T}$. Exclusion criteria were incomplete examinations $(n=6)$, previous treatment $(n=8)$, and tumor of the contralateral breast (BI-RADS 2-5) $(n=36)$. All lesions were histopathologically verified after ${ }^{18} \mathrm{~F}-\mathrm{FDG}$ PET/CT and MRI by surgical or image-guided biopsy. Thus, 141 patients (140 female; mean age, $57 \pm$ $14.3 \mathrm{y}$; range, $18-86 \mathrm{y}$ ) with a tumor-free contralateral breast on mammography, ultrasound, MRI, and ${ }^{18} \mathrm{~F}-\mathrm{FDG}$ PET/MRI (BI-RADS 1) were included in this retrospective analysis. Several patients have been previously analyzed and reported in a different context $(17,19,22)$.

\section{Imaging}

All patients underwent combined multiparametric ${ }^{18} \mathrm{~F}-\mathrm{FDG}$ PET/MRI with ${ }^{18} \mathrm{~F}$-FDG PET/CT and 3-T multiparametric MRI of the breast. Examinations were no longer than $6 \mathrm{~d}$ apart (mean, 1.15; range, 1-6; same day, $n=70 ; 1 \mathrm{~d}, n=31 ; 2 \mathrm{~d}, n=12 ; 3 \mathrm{~d}, n=11 ; 4 \mathrm{~d}, n=11 ; 5 \mathrm{~d}, n=$ $5 ; 6 \mathrm{~d}, n=1)$.

\section{${ }^{18}$ F-FDG PET/CT}

PET imaging was performed using a combined PET/CT in-line system (Biograph 64 TruePoint PET/CT system; Siemens). Patients fasted $6 \mathrm{~h}$ before the body weight-adapted injection of approximately $300 \mathrm{MBq}$ of ${ }^{18} \mathrm{~F}-\mathrm{FDG}$. Blood glucose levels were less than $150 \mathrm{mg} / \mathrm{dL}$ $(8.3 \mathrm{mmol} / \mathrm{L})$. Scanning started after an uptake time of $45 \mathrm{~min}$. CT images were used for attenuation correction. PET images were reconstructed using the iterative TrueX algorithm (Siemens). Four iterations per 21 subsets were used, with a matrix size of $168 \times 168$, transaxial field of view (FOV) of $605 \mathrm{~mm}$ (pixel size, $3.6 \mathrm{~mm}$ ), and section thickness of $5 \mathrm{~mm}$.

\section{Multiparametric MRI}

MRI was performed using a 3-T MRI scanner (Tim Trio; Siemens) and a 4-channel breast coil (InVivo) with the patient prone. In premenopausal women, MRI was performed between days 7 and 14 of the menstrual cycle (4). The MRI protocol consisted of a fat-saturated T2weighted turbo spin-echo sequence, with a repetition time/echo time (TR/TE) of 4,800/9 ms, FOV of $340 \mathrm{~mm}, 48$ slices at $3 \mathrm{~mm}$, flip angle of $128^{\circ}, 384 \times 512$ matrix, and acquisition time of $2 \min 16 \mathrm{~s}$.

The protocol also consisted of axial 3-acquisition trace diffusionweighted, double-refocused, single-shot echo-planar imaging with inversion recovery fat suppression (TR/TE, $8,000 / 59 \mathrm{~ms}$; inversion time, $210 \mathrm{~ms}$; FOV, $360 \times 202 \mathrm{~mm}$; 24 slices at $5 \mathrm{~mm}$; intersection gap, 10\%; matrix, $172 \times 96$ [50\% oversampling]; b-values, 50 and $850 \mathrm{~s} / \mathrm{mm}$; acquisition time, 2 min $56 \mathrm{~s}$ ) (23).

For dynamic contrast-enhanced MRI until December 2011, a hybrid protocol was used (24) consisting of 5 alternating sections of highspatial-resolution and high-temporal-resolution T1-weighted sequences using 3-dimensional turbo fast low-angle shot without preparation pulse and with selective water excitation (TR/TE, 877/3.82 ms; FOV, $320 \mathrm{~mm}$; 96 slices; 1-mm isotropic resolution; matrix, $320 \times 134$; 1 average; bandwidth, $200 \mathrm{~Hz} /$ pixel) and a T1-weighted volume-interpolated breathhold examination (TR/TE, 3.61/1.4 ms; FOV, 320 mm; 72 slices; 1.7-mm isotropic resolution; matrix, $192 \times 192$; 1 average; bandwidth, $400 \mathrm{~Hz} /$ pixel; acquisition time, 9 min $20 \mathrm{~s}$ ).

From January 2012 onward, transversal T1-weighted time-resolved angiography with stochastic trajectories was performed (water excitation fat-saturation; TR/TE, $6.23 / 2.95 \mathrm{~ms}$; flip angle, $15^{\circ}$; FOV, $196 \times 330 \mathrm{~mm}$; 144 slices; spatial resolution, $0.9 \times 0.9 \times 1 \mathrm{~mm}$; temporal interpolation factor, 2; temporal resolution, $14 \mathrm{~s}$; matrix, $384 \times 384$; 1 average; center $\mathrm{k}$-space region; resampling rate, $23 \%$; reacquisition density peripheral k-space, 20\%; acquisition time, 6 min $49 \mathrm{~s}$ ).

A standard dose $(0.1 \mathrm{mmol} / \mathrm{kg}$ of body weight $)$ of gadoterate meglumine (Dotarem; Guerbet) was injected in an antecubital vein using a power injector (Spectris Solaris EP; Medrad) at $4 \mathrm{~mL} / \mathrm{s}$, followed by a saline flush.

To generate combined ${ }^{18} \mathrm{~F}$-FDG PET/MRI data, multiparametric MRI and PET data were fused semiautomatically using the landmarkmatching tool of the TrueD fusion workstation (Siemens).

\section{Data Analysis}

In all patients, 2 readers ( $\mathrm{r} 1$ and $\mathrm{r} 2 ; 13$ and $4 \mathrm{y}$ of experience, respectively) independently assessed the following imaging biomarkers from the contralateral tumor-free breast: BPU (from ${ }^{18} \mathrm{~F}-\mathrm{FDG}$ PET), FGT (from unenhanced fat-saturated T1-weighted sequences), BPE (from dynamic contrast-enhanced MRI on early postcontrast sequences), and mean $\mathrm{ADC}$ (from diffusion-weighted imaging).

For quantification of BPU from ${ }^{18} \mathrm{~F}$-FDG PET, a 3-dimensional volume of interest was placed around the parenchyma of the normal contralateral breast by a breast radiologist trained in hybrid imaging, under the supervision of a nuclear medicine physician, using the TrueD workstation (Siemens). Adequate distance from surrounding anatomic structures was maintained. The $\mathrm{SUV}_{\max }$ was recorded from each volume of interest. Measurements were repeated 3 times and averaged. The same reader $(\mathrm{r} 1)$ repeated all $\mathrm{SUV}_{\text {max }}$ measurements $3 \mathrm{wk}$ later to calculate intrareader agreement. Another independent reader (r2) repeated all measurements to assess interreader agreement.

BPE and FGT in MRI of the healthy contralateral breast were assessed qualitatively by 2 breast radiologists independently ( $\mathrm{r} 1, \mathrm{r} 2)$. As recommended in the revised ACR BI-RADS MRI lexicon (25), FGT and BPE were evaluated through visual subjective estimation. FGT was classified as ACR a for almost entirely fatty breasts, ACR b for scattered FGT, ACR $\mathrm{c}$ for heterogeneous FGT, and ACR d for breasts with extreme amounts of FGT. BPE was graded as minimal, mild, moderate, or marked. Interreader agreement was calculated for both parameters.

To assess mean ADCs, a region of interest was drawn manually on the healthy contralateral breast parenchyma on ADC maps by 2 breast radiologists independently $(\mathrm{r} 1, \mathrm{r} 2)$. 


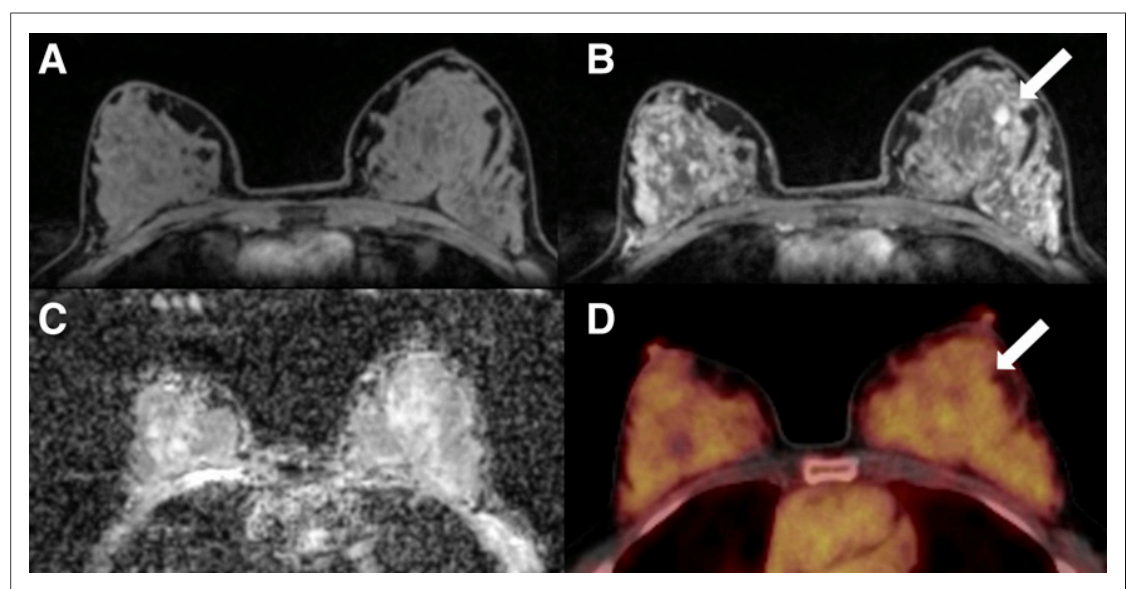

FIGURE 1. A 50-y-old postmenopausal woman with fibroadenoma (arrows) in left breast. (A) Unenhanced fat-saturated T1-weighted MRI shows extreme amount of FGT (ACR d). (B) Moderate $\mathrm{BPE}$ is seen on dynamic contrast-enhanced MRI at $90 \mathrm{~s}$. (C) Mean ADC of breast parenchyma of contralateral breast on diffusion-weighted imaging with ADC mapping is $1.5 \times 10^{-3} \mathrm{~mm}^{2} / \mathrm{s}$. (D) On ${ }^{18} \mathrm{~F}-\mathrm{FDG}$ PET/CT, lesion is not ${ }^{18} \mathrm{~F}-\mathrm{FDG}$-avid, and BPU of normal breast parenchyma is relatively high, with $\mathrm{SUV}_{\max }$ of 3.2 .

BPU, BPE, and FGT of the ipsilateral diseased breast were also assessed.

\section{Statistical Analysis}

Descriptive statistics were used to summarize continuous variables, frequencies, and percentages. The association between disease status (malignant/benign) and imaging parameters was evaluated using the Fisher exact test for categoric variables and the Wilcoxon rank-sum test for continuous variables. The Wilcoxon rank-sum test was also applied to compare BPE, BPU, and FGT between the healthy and affected breast.

A stratified analysis was conducted to test confounding by menopause.

Inter- and intrareader agreement were assessed using the concordance correlation coefficient; the closer the value is to 1 , the better the agreement (26).

We considered $P$ values of less than 0.05 as statistically significant. Statistical analyses were conducted using SAS release 9.4 (SAS Institute).

\section{RESULTS}

There were 100 malignant (mean size, $27 \mathrm{~mm}$ [range, 6-100 $\mathrm{mm}$ ]) and 41 benign (mean size, $23 \mathrm{~mm}$ [range, 5-80 mm]) lesions. Of the patients with malignant breast tumors, 20 were premenopausal (20\%), 2 perimenopausal (2\%), and 78 postmenopausal (78\%), whereas of the patients with benign lesions, 19 were premenopausal $(46.3 \%), 1$ perimenopausal $(2.4 \%)$, and 21 postmenopausal $(51.2 \%)$.

Mean, SD, minimum, and maximum BPU $\mathrm{SUV}_{\max }$ of healthy contralateral breast tissue on ${ }^{18} \mathrm{~F}-\mathrm{FDG}$ PET/CT was 1.8, 0.6, 0.9, and 4.6, respectively. BPU differed significantly between patients with benign (mean, 1.9) and malignant (mean, 1.8) lesions $(P<$ 0.001) (Fig. 1).

The results of the ACR classification for BPE and FGT by both readers are summarized in Table 1 . BPE differed significantly $(P<0.001)$ between patients with benign and malignant lesions, with patients with cancer demonstrating a lower BPE in the contralateral breast (Fig. 2). FGT between patients with benign and malignant lesions approached but did not achieve statistical significance
$(P=0.055)$. Mean BPU $\mathrm{SUV}_{\max }$ and SD was $1.5 \pm 0.6$ for patients with minimal BPE, $1.9 \pm 0.6$ for mild BPE, $2.2 \pm 0.5$ for moderate BPE, and $1.9 \pm 0.8$ for marked BPE. Mean BPU SUV $\max$ and SD was $1.5 \pm$ 0.5 for patients with ACR a, $1.7 \pm 0.5$ for ACR b, $2.1 \pm 0.7$ for ACR c, and $2.5 \pm 0.6$ for ACR d. Results are based on $\mathrm{r} 1$.

Mean, SD, minimum, and maximum mean $\mathrm{ADC}$ of healthy contralateral breast tissue on diffusion-weighted imaging were $1.72,0.27,1.12$, and $2.4 \times 10^{-3} \mathrm{~mm}^{2} / \mathrm{s}$, respectively. Mean ADC did not differ significantly between benign (mean, $1.7 \times 10^{-3}$ $\mathrm{mm}^{2} / \mathrm{s}$ ) and malignant (mean, $1.74 \times 10^{-3}$ $\left.\mathrm{mm}^{2} / \mathrm{s}\right)$ lesions $(P=0.19)$.

The crude odds radio for the association between menopause and breast cancer was assessed. Menopause was not associated with outcome among the unexposed (odds radio, 3.64; 95\% confidence interval, 0.2945.95 ) and the exposed (odds radio, 0.06; 95\% confidence interval, 0.018-0.219). Controlling for menopause changed the results less than $10 \%$.

There were no significant differences in imaging biomarkers between contralateral healthy and ipsilateral diseased breast, excluding a potential stealing phenomenon of the diseased breast with respect to vascularity and metabolic activity.

Mean scores among patients with malignant lesions for BPE of the affected and healthy breast were similar ( $\mathrm{r} 1$ range, 60.4-62.9; r2 range, 60.1-62.6). Likewise, mean scores among patients with malignant lesions for BPU and FGT of the affected and healthy breast were similar (BPU: r1 range, 61.4-65.5; r2 range, 62.6-65.5; FGT: r1 range, 63.6-66; r2 range, 63.7-66.9). Similarly, in patients with benign lesions, no asymmetry in BPE, BPU, and FGT was found.

Inter- and intrareader agreement did not vary considerably among most parameters (Table 2). The best result was achieved for intrareader agreement of BPU (concordance correlation coefficient, 0.96), followed by interreader agreement of BPU (concordance correlation coefficient,

\section{TABLE 1}

ACR Classification for BPE and FGT by Both Readers

\begin{tabular}{lcl}
\hline \multicolumn{1}{l}{ Imaging characteristic } & $\mathrm{r} 1$ & $\mathrm{r} 2$ \\
\hline BPE & & \\
\hline Minimal & $61(43.3 \%)$ & $65(46.1 \%)$ \\
\hline Mild & $56(39.7 \%)$ & $52(36.9 \%)$ \\
\hline Moderate & $19(13.5 \%)$ & $17(12.1 \%)$ \\
\hline Marked & $5(3.5 \%)$ & $7(5 \%)$ \\
FGT & & \\
Almost entirely fat & $35(24.8 \%)$ & $33(23.4 \%)$ \\
\hline Scattered fibroglandular & $61(43.3 \%)$ & $64(45.4 \%)$ \\
Heterogeneously dense & $29(20.6 \%)$ & $24(17 \%)$ \\
\hline Extremely dense & $16(11.3 \%)$ & $20(14.2 \%)$ \\
\end{tabular}

Data are numbers of subjects, with percentages in parentheses. 


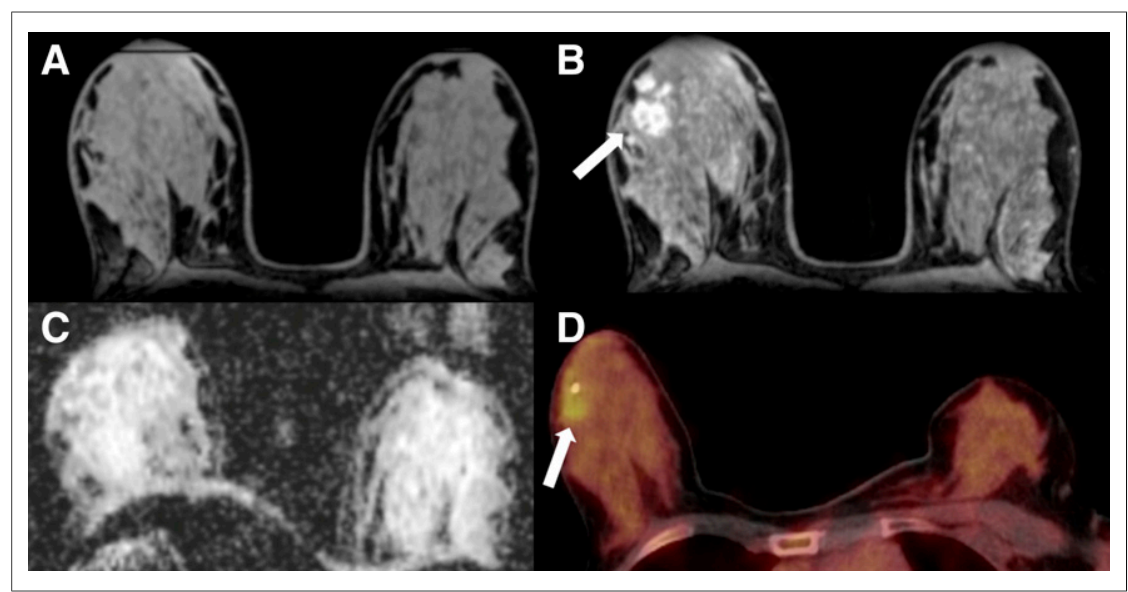

FIGURE 2. Mucinous carcinoma (arrows) in right breast in 42-y-old premenopausal woman. (A) Precontrast fat-saturated T1-weighted MR images show extreme amount of FGT (ACR d). (B) BPE in dynamic contrast-enhanced MRI at $90 \mathrm{~s}$ is mild. (C) On diffusion-weighted imaging, ADCs of normal breast parenchyma are $2.17 \times 10^{-3} \mathrm{~mm}^{2} / \mathrm{s}$. (D) SUV $\max (B P U)$ in ${ }^{18} \mathrm{~F}-\mathrm{FDG}$ PET/CT is 2.58 .

0.95). Agreement was substantial for all parameters, except interreader agreement of mean $\mathrm{ADC}$, when compared with the others.

\section{DISCUSSION}

Hybrid PET/MRI scanners are now being used in clinical practice (19-21) and can simultaneously assess and spatiolongitudinally monitor different PET and MRI biomarkers. In this study, we demonstrate differences in multiparametric ${ }^{18} \mathrm{~F}-\mathrm{FDG}$ PET/MRI biomarkers, obtained from contralateral healthy breast tissue, between patients with benign and patients with malignant breast tumors. Contralateral BPU, BPE, and, to a lesser degree, FGT are lower in patients with a breast malignancy; hence, they may be useful biomarkers for the presence of cancer, with the potential to significantly contribute to risk-adapted screening and risk-reduction strategies in clinical practice. However, there are no differences in the ADCs of contralateral breast parenchyma between patients with benign and patients with malignant lesions.

To our knowledge, this is the first study using ${ }^{18} \mathrm{~F}-\mathrm{FDG}$ PET to examine patients with benign and malignant tumors for differences in BPU of the contralateral tumor-free breast. In contrast to both BPE and FGT, which are usually assessed through subjective visual estimation, BPU can be easily quantified, is highly reproducible $(17,27,28)$, and therefore may serve as a more stable, noninvasive imaging biomarker. In a recent study that assessed the

TABLE 2

Inter- and Intrareader Agreement of Parameters of Healthy Contralateral Breast

\begin{tabular}{lc}
\hline Agreement & Concordance correlation coefficient \\
\hline Intrareader BPU & $0.956(0.942,0.970)$ \\
\hline Interreader BPU & $0.949(0.932,0.965)$ \\
Interreader BPE & $0.907(0.878,0.937)$ \\
Interreader FGT & $0.933(0.911,0.954)$ \\
Interreader mean ADC & $0.677(0.587,0.766)$ \\
\hline
\end{tabular}

95\% confidence intervals are given in parentheses. correlation and reproducibility of quantitatively measured BPU with qualitatively evaluated BPE and FGT as well as age, there were significant direct correlations between BPU and BPE, and between BPU and FGT, of the healthy contralateral breast (17), with almost perfect inter- and intrarater agreement for all parameters. These results were confirmed by Mema et al., who performed qualitative and quantitative analysis of BPE (18), and by An et al., who demonstrated a significant direct correlation of BPU with BPE (29).

In the present study, we found that BPE of the contralateral tumor-free breast was significantly lower in patients with breast cancer. These findings are unexpected, as previous studies found that breast cancer risk increases with higher levels of BPE $(11,13)$. These results were confirmed by Grimm et al., who compared 61 high-risk breast cancer patients with high-risk controls matched for age and high-risk indication who did not develop cancer (30). An interpretation of these findings is that BPE represents the metabolic activity of breast tissue and, as such, a favorable environment for cancer development (31). Nevertheless, it must be noted that the biologic parameter that BPE truly represents has not yet been discovered. In this study, we investigated differences in patients with benign and malignant breast tumors at average risk of breast cancer. Our divergent results with respect to BPE might be explained by the fact that prior studies focused solely on a high-risk population with and without the development of breast cancer, whose breast tissue is known to differ substantially from women of average cancer risk (32). Additionally, in the first 2 studies, there is no information available on the time point of MRI examinations during menstrual cycle, and in the study by Dontchos et al. (13), the proportion of postmenopausal women is unclear. However, it has to be noted that so far differences in BPE and BPU of the contralateral unaffected breast in high-risk women with benign and malignant lesions have not been investigated. If our findings can be confirmed also in this patient collective, there might be relevant clinical applications. If there is a longitudinal decrease of BPE and BPU without concurrent development of a suggestive MRI finding, short-term follow-up might be considered to facilitate detection of an arising breast cancer at the earliest stage. If there is a longitudinal decrease of BPE and BPU with concurrent development of a MRI finding, our results indicate the necessity for image-guided breast biopsy even if the lesion presents with probably-benign imaging features (BI-RADS 3). Bennani-Baiti et al., the first to investigate BPE in an average-risk population, found no association between breast cancer odds and BPE (33). To examine a potential stealing phenomenon of contrast agent/tracer to the breast with a malignancy due to increased vascularity, we additionally evaluated BPE and BPU of the ipsilateral diseased breast with FGT assessed as a reference. We found neither a left-right asymmetry of FGT in patients with malignant and benign lesions nor significant differences in BPE and BPU between contralateral healthy and ipsilateral diseased breast, excluding a stealing phenomenon of the diseased breast. At this point, the underlying processes for the results of our study remain unclear and must be confirmed by studies with larger numbers of individuals at average cancer risk.

Although FGT in MRI is equivalent to mammographic breast density - an established independent risk factor for breast cancer (31) - the role of FGT with regard to breast cancer risk remains 
unclear. Although King et al. demonstrated mildly increased breast cancer odds for patients with increased FGT (odds radio, 1.2) in a high-risk population (11), Bennani-Baiti et al. found no correlation between FGT and cancer risk in an average-risk population (33). In the present study, contralateral FGT was found to be decreased in patients with breast cancer, although the difference was not significant. Further research is warranted to explore the potential of FGT as an imaging biomarker for breast cancer.

Although studies that investigate diffusion-weighted imaging of healthy breast parenchyma are rare, McDonald et al. showed that breast tissue ADCs increase with mammographic breast density but are independent of BPE (34). Other previous studies have demonstrated stable ADCs of normal breast parenchyma during different phases of the menstrual cycle $(35,36)$. A recent study found that the ADCs of 248 benign and malignant lesions were independent of BPE, FGT, and menopausal status (37). These findings are in good agreement with our results, in which ADCs did not differ significantly between patients with benign and patients with malignant breast lesions.

This study has limitations. BPE and FGT were assessed qualitatively; the ACR BI-RADS lexicon currently does not recommend quantitative measurements of those parameters, and no standardized measurement tool is available (25). However, excellent inter- and intrareader agreement for BPE and FGT was demonstrated previously (17). Second, the fact that not all PET/CT and MRI examinations were performed on the same day might have affected BPU and BPE because of hormonal changes. Nevertheless, the time between the 2 examinations was short (mean, $1.15 \mathrm{~d}$ ); hence, substantial changes in BPU and BPE should not have occurred. Third, it might be possible that extensive ${ }^{18} \mathrm{~F}-\mathrm{FDG}$ avidity of a large tumor might falsely decrease ${ }^{18}$ F-FDG uptake in the contralateral breast. Nevertheless, we did not find a side difference in BPU between the healthy and the affected breast in all patients. Additionally, as PET/CT is an excellent tool for the detection of distant metastasis, and the mean tumor sizes of benign and malignant lesions in our patient collective were similar ( 23 and $27 \mathrm{~mm}$, respectively), an impact seems unlikely. Further studies with bilateral quantitative assessment of ${ }^{18} \mathrm{~F}-\mathrm{FDG}$ PET/MRI biomarkers are warranted to confirm our findings.

\section{CONCLUSION}

Differences in multiparametric ${ }^{18} \mathrm{~F}$-FDG PET/MRI biomarkers, obtained from contralateral tumor-free breast tissue, exist between patients with benign and patients with malignant breast tumors. Contralateral BPE, BPU, and FGT are decreased in breast cancer patients and may potentially serve as imaging biomarkers for the presence and risk of malignancy.

\section{DISCLOSURE}

Funding was provided by the 2020 Research and Innovation Framework Programme PHC-11-2015 number 667211-2, seed grants from Novomed Austria and Guerbet France, the National Institutes of Health/National Cancer Institute Cancer Center Support Grant P30 CA008748, Breast Cancer Research Foundation, and Susan G. Komen Foundation. Maxine S. Jochelson received speaker honoraria from GE Healthcare. No other potential conflict of interest relevant to this article was reported.

\section{ACKNOWLEDGMENTS}

We gratefully acknowledge the support in manuscript writing and editing from Joanne Chin.
KEY POINTS

QUESTION: The aim of this study was to evaluate whether there are differences in ${ }^{18} \mathrm{~F}-\mathrm{FDG}$ PET/MRI biomarkers of contralateral healthy breast tissue between patients with benign and patients with malignant breast lesions.

PERTINENT FINDINGS: In this retrospective study including 141 patients, a significant difference in BPE and BPU between patients with benign and patients with malignant lesions was found. Patients with cancer showed lower BPE and BPU in the tumor-free breast.

IMPLICATIONS FOR PATIENT CARE: Imaging features of the contralateral breast may potentially serve as biomarkers for the risk and presence of malignancy.

\section{REFERENCES}

1. Hamstra DA, Rehemtulla A, Ross BD. Diffusion magnetic resonance imaging: a biomarker for treatment response in oncology. J Clin Oncol. 2007;25:4104-4109.

2. Tabár L, Vitak B, Chen TH, et al. Swedish two-county trial: impact of mammographic screening on breast cancer mortality during 3 decades. Radiology. 2011;260:658-663.

3. Pisano ED, Gatsonis C, Hendrick E, et al. Diagnostic performance of digital versus film mammography for breast-cancer screening. $N$ Engl J Med. 2005;353: 1773-1783.

4. Mann RM, Balleyguier C, Baltzer PA, et al. Breast MRI: EUSOBI recommendations for women's information. Eur Radiol. 2015;25:3669-3678.

5. Sardanelli F, Boetes C, Borisch B, et al. Magnetic resonance imaging of the breast: recommendations from the EUSOMA working group. Eur J Cancer. 2010; 46:1296-1316.

6. Saslow D, Boetes C, Burke W, et al. American Cancer Society guidelines for breast screening with MRI as an adjunct to mammography. CA Cancer J Clin. 2007;57:75-89.

7. Monticciolo DL, Newell MS, Moy L, Niell B, Monsees B, Sickles EA. Breast cancer screening in women at higher-than-average risk: recommendations from the ACR. J Am Coll Radiol. 2018;15:408-414.

8. Kuhl CK, Strobel K, Bieling H, Leutner C, Schild HH, Schrading S. Supplemental breast MR imaging screening of women with average risk of breast cancer. Radiology. 2017;283:361-370.

9. Amarosa AR, McKellop J, Klautau Leite AP, et al. Evaluation of the kinetic properties of background parenchymal enhancement throughout the phases of the menstrual cycle. Radiology. 2013;268:356-365.

10. Baltzer PA, Dietzel M, Vag T, et al. Clinical MR mammography: impact of hormonal status on background enhancement and diagnostic accuracy. Rofo. 2011; 183:441-447.

11. King V, Brooks JD, Bernstein JL, Reiner AS, Pike MC, Morris EA. Background parenchymal enhancement at breast MR imaging and breast cancer risk. Radiology. 2011;260:50-60.

12. Boyd NF, Guo H, Martin LJ, et al. Mammographic density and the risk and detection of breast cancer. $N$ Engl J Med. 2007;356:227-236.

13. Dontchos BN, Rahbar H, Partridge SC, et al. Are qualitative assessments of background parenchymal enhancement, amount of fibroglandular tissue on MR images, and mammographic density associated with breast cancer risk? Radiology. 2015;276:371-380.

14. Pinker K, Bickel H, Helbich TH, et al. Combined contrast-enhanced magnetic resonance and diffusion-weighted imaging reading adapted to the "Breast Imaging Reporting and Data System" for multiparametric 3-T imaging of breast lesions. Eur Radiol. 2013;23:1791-1802.

15. Martincich L, Deantoni V, Bertotto I, et al. Correlations between diffusionweighted imaging and breast cancer biomarkers. Eur Radiol. 2012;22:1519-1528.

16. Bickel H, Pinker-Domenig K, Bogner W, et al. Quantitative apparent diffusion coefficient as a noninvasive imaging biomarker for the differentiation of invasive breast cancer and ductal carcinoma in situ. Invest Radiol. 2015;50:95-100.

17. Leithner D, Baltzer PA, Magometschnigg HF, et al. Quantitative assessment of breast parenchymal uptake on ${ }^{18} \mathrm{~F}$-FDG PET/CT: correlation with age, background parenchymal enhancement, and amount of fibroglandular tissue on MRI. J Nucl Med. 2016;57:1518-1522.

18. Mema E, Mango VL, Guo X, et al. Does breast MRI background parenchymal enhancement indicate metabolic activity? Qualitative and 3D quantitative computer imaging analysis. J Magn Reson Imaging. 2018;47:753-759. 
19. Magometschnigg HF, Baltzer PA, Fueger B, et al. Diagnostic accuracy of ${ }^{18} \mathrm{~F}-$ FDG PET/CT compared with that of contrast-enhanced MRI of the breast at $3 \mathrm{~T}$. Eur J Nucl Med Mol Imaging. 2015;42:1656-1665.

20. Avril S, Muzic RF Jr, Plecha D, Traughber BJ, Vinayak S, Avril N. ${ }^{18}$ F-FDG $\mathrm{PET} / \mathrm{CT}$ for monitoring of treatment response in breast cancer. J Nucl Med. 2016; 57(suppl 1):34S-39S.

21. Koolen BB, van der Leij F, Vogel WV, et al. Accuracy of ${ }^{18} \mathrm{~F}-\mathrm{FDG}$ PET/CT for primary tumor visualization and staging in T1 breast cancer. Acta Oncol. 2014;53:50-57.

22. Pinker K, Bogner W, Baltzer P, et al. Improved differentiation of benign and malignant breast tumors with multiparametric ${ }^{18}$ fluorodeoxyglucose positron emission tomography magnetic resonance imaging: a feasibility study. Clin Cancer Res. 2014;20:3540-3549.

23. Bogner W, Pinker-Domenig K, Bickel H, et al. Readout-segmented echo-planar imaging improves the diagnostic performance of diffusion-weighted MR breast examinations at 3.0 T. Radiology. 2012;263:64-76.

24. Pinker K, Grabner G, Bogner W, et al. A combined high temporal and high spatial resolution 3 tesla MR imaging protocol for the assessment of breast lesions: initial results. Invest Radiol. 2009;44:553-558.

25. D'Orsi CJSE, Mendelson EB, Morris EA, et al. ACR BI-RADS® Atlas, Breast Imaging Reporting and Data System. Reston, VA: American College of Radiology; 2013.

26. Lin LI. A concordance correlation coefficient to evaluate reproducibility. Biometrics. 1989;45:255-268.

27. Vranjesevic D, Schiepers C, Silverman DH, et al. Relationship between ${ }^{18}$ F-FDG uptake and breast density in women with normal breast tissue. J Nucl Med. 2003; 44:1238-1242.

28. Mavi A, Cermik TF, Urhan M, et al. The effect of age, menopausal state, and breast density on ${ }^{18} \mathrm{~F}$-FDG uptake in normal glandular breast tissue. J Nucl Med. 2010;51:347-352.
29. An YS, Jung Y, Kim JY, et al. Metabolic activity of normal glandular tissue on ${ }^{18}$ F-fluorodeoxyglucose positron emission tomography/computed tomography: correlation with menstrual cycles and parenchymal enhancements. J Breast Cancer. 2017;20:386-392.

30. Grimm LJ, Saha A, Ghate SV, et al. Relationship between background parenchymal enhancement on high-risk screening MRI and future breast cancer risk. Acad Radiol. 2019;26:69-75.

31. Pike MC, Pearce CL. Mammographic density, MRI background parenchymal enhancement and breast cancer risk.Ann Oncol. 2013;24(suppl 8):viii37-viii41.

32. Ramadan S, Arm J, Silcock J, et al. Lipid and metabolite deregulation in the breast tissue of women carrying BRCA1 and BRCA2 genetic mutations. Radiology. 2015;275:675-682.

33. Bennani-Baiti B, Dietzel M, Baltzer PA. MRI background parenchymal enhancement is not associated with breast cancer. PLoS One. 2016;11:e0158573.

34. McDonald ES, Schopp JG, Peacock S, et al. Diffusion-weighted MRI: association between patient characteristics and apparent diffusion coefficients of normal breast fibroglandular tissue at 3 T. AJR. 2014;202:W496-W502.

35. Partridge SC, McKinnon GC, Henry RG, Hylton NM. Menstrual cycle variation of apparent diffusion coefficients measured in the normal breast using MRI. J Magn Reson Imaging. 2001;14:433-438.

36. O'Flynn EA, Morgan VA, Giles SL, deSouza NM. Diffusion weighted imaging of the normal breast: reproducibility of apparent diffusion coefficient measurements and variation with menstrual cycle and menopausal status. Eur Radiol. 2012;22:1512-1518.

37. Horvat JV, Durando M, Milans S, et al. Apparent diffusion coefficient mapping using diffusion-weighted MRI: impact of background parenchymal enhancement, amount of fibroglandular tissue and menopausal status on breast cancer diagnosis. Eur Radiol. 2018;28:2516-2524. 